\title{
Determinants of Information Systems Adoption and Deployment in SMEs: A Case Study of Micro Finance Institutions in TransNzoia County, Kenya
}

\author{
Peter Namisiko \\ School of Pure and Applied Sciences \\ Mount Kenya University, Kitale Campus \\ P.O BOX 1869-30200, Kitale
}

\author{
Robert Mindila \\ School of Business and Public Management \\ Mount Kenya University, Kitale Campus \\ P.O BOX 1869-30200, Kitale
}

\begin{abstract}
Information Technology has become a vital and integral part of every business in the World. IT is one of the strongest drivers for competitive, innovation and change in modern business environment. The SME sector in Kenya employs 80 percent of the total population and contributes over 40 percent to GDP. Majority of people employed in this sector is the youthful population. However, adoption and deployment of Information Technology in this sector has not been satisfactory compared to large corporations. This paper examined the Determinants to Adoption and Deployment of Information Systems in this sector. A quantitative Descriptive Survey Design was used in this Study. Data was collected from 3 SMEs in TransNzoia County using interviews and Questionnaire. Results obtained from this study shows that CEOs characteristics, IS characteristics, Organization's characteristics and Environmental Characteristics play a significant role in determining whether IS will be adopted and deployed in the SMEs. It is hoped that the findings from this study will enable policy makers in the ICT sector formulate proper legislative framework that informs how well ICT can be harnessed for the growth of the SME sector in Kenya.
\end{abstract}

\section{General Terms}

Determinants of IS Adoption by SMEs

\section{Keywords}

Information Systems, Technology, Adoption, Deployment. SMEs, TransNzoia County

\section{INTRODUCTION}

According to (Thong, 1999), technological adoption in any organization undergoes three phases namely: Initiation phase, Adoption phase and Implementation phase. The initiation phase involves elicitation, collection and analysis of information needs of an organization. This is followed by the adoption stage in which a decision is made about adopting the technological innovation. When the decision is to go ahead with adoption, the implementation stage involves implementing the technological innovation in the business. Information Technology has become a vital and integral part of every business in the World. IT is one of the strongest drivers for competitive, innovation and change in modern business environment. Effective deployment and adoption of Information Technology in an organization may lead to efficiency and make an organization have a competitive advantage (Barkley, Markley, \& Lamie, 2008). The SME sector in Kenya employs 80 percent of the total population and contributes over 40 percent to GDP. Majority of people employed in this sector is the youthful population. However, adoption and deployment of Information Technology in this sector has not been satisfactory compared to large corporations(Dien, 2002). According to (Thong, 1999) there is a major shift on how organizations have been using Information Technology. While large corporation businesses have been using Information Technology for some time, small businesses have been slow in adopting these technological innovations. This slow adoption rate is a critical issue, since small businesses constitute over 90 percent of all businesses in many countries especially the developing ones. For instance, the SME sector in Kenya employs 80 percent of the total population and contributes over 40 percent to GDP. Deployment and adoption of Information Systems is critical to the growth of this sector.

Literature available on the Adoption of Information systems shows that majority of research has concentrated on implementation phase of technological adoption (Thong, 1999). According to this school of thought, the initiation stage and adoption stage of technological deployment and adoption have been conceptualized well by organizations both large corporations and SMEs. Majority of research in this area also shows that determinants of information system adoption by SMEs involve three main characteristics namely: The CEO Characteristics, Organization Characteristics, Information System Characteristics and Environmental Characteristics(TS Tran, 2011). Other studies have found that attitude, subjective norms, and perceived control are antecedents of intentions to adopt Information Systems in an organization (S Jackson, 2011). According to this school of thought, deployment and adoption of information system in an organization is likely to be influenced by attitudes of employees in that organization, organization norms and culture and perceived control of Information System which include level of understanding in terms of usage. The difference in Information Systems adoption by large corporations and SMEs may be attributed to the differences in these two business entities. According to (Thong, 1999), highly centralized structures, tendency to employ generalists rather than specialists and financial resources makes SMEs to be unique. For instance, if SMEs wanted to recruit IS specialists, they face difficulties in 
attracting and retaining skilled IS staff because of the limited career paths available in a small business. As a result of this scenario, there is a lower level of awareness of the benefits of Information Systems and a lack of Information System knowledge and technical skill in SMEs. This hinders Information System adoption. In addition, small businesses lack financial resources and are highly susceptible to shortrange planning in response to their highly competitive environment. However, large corporations are different from the SMEs in terms of Information System adoption since they have decentralized management structures, recruit highly specialized skilled manpower and have necessary resources to deploy and adopt Information Systems.

Due to these unique characteristics of SMEs, there is a need to study the critical determinants of information system adoption in these organizations. Various studies have been conducted to ascertain the determinants of Information System Adoption in different countries. For instance, a study conducted by (TS Tran, 2011) found that Information System characteristics play a significant role in the adoption of technology in an organization. Another study conducted by Thong (Thong, 1999) in Singapore found that Organization Characteristics, Information System Characteristics, CEO characteristics and Environmental characteristics play an important role in determining Information System adoption within an organization. This is informed by the fact that determinants of Information System adoption in one country may not be necessarily the same determinants in another country due to the differences in regulatory framework, level of ICT infrastructure in that country and general understanding of IT and Knowledge (Namisiko \& Aballo, 2013). Currently, there is no study that has been conducted in TransNzoia County to ascertain the critical determinants of Information System adoption. With devolved government structures in Kenya, it is critical that the policy stakeholders in different counties understand what informs employees in an organization to adopt the Information technology if the country is to attain the goals and objectives of Kenya Vision 2013.

The main objective of this study was to evaluate the determinants of Information Systems adoption and deployment by SMEs in TransNzoia County. Specifically the study intended:

a) To evaluate the determinants of Information System Adoption and deployment by SMEs in TransNzoia County, Kenya

b) To evaluate the challenges facing the deployment and Adoption of Information Systems by SMEs in TransNzoia County, Kenya

This study was informed by the fact that the country's economic blue print, the Kenya Vision 2030, has identified ICT as a key enabler to the attainment of the goals and aspirations of the Kenya Vision 2030. The thrust of the Vision in the ICT Sector is therefore to convert Kenya into a truly knowledge and information economy by enabling access to quality, affordable and reliable ICT services in the country (CCK, 2010). With devolved government structures in Kenya, it is critical that the policy stakeholders in different counties understand what informs employees in an organization to adopt the Information technology if the country is to attain the goals and objectives of Kenya Vision 2030. The paper has four parts. First, it reviewed the literature related to Information Systems and adoption. Then, research methodology is presented and data analysis techniques are discussed. Next, the findings are discussed and summarised. The paper concludes with recommendations on Information System adoption by SMEs in TransNzoia County.

\section{LITERATURE REVIEW 2.1 Theoretical Framework}

The theoretical framework adopted in this study is Diffusion of Innovations Theory. Diffusion of Innovations is a theory that seeks to explain how, why, and at what rate new ideas and technology spread through cultures (Sahin I, 2005). According to Rogers, Innovation attributes supporting diffusion are: Relative advantage, compatibility, complexity, observability and trialibility. In this study, Information System Characteristics such as Relative advantage, compatibility, complexity and trialibility for innovation and IS Adoption. Rogers categorized innovations into two types: preventive and incremental (non-preventive) innovations. A preventive innovation is a new idea that an individual adopts now in order to lower the probability of some unwanted event. Preventive innovations usually have a slow rate of adoption so their relative advantage is highly uncertain. However, incremental innovations provide beneficial outcomes in a short period. Based on this theory, the following conceptual framework was derived. 


\subsection{Conceptual Framework}

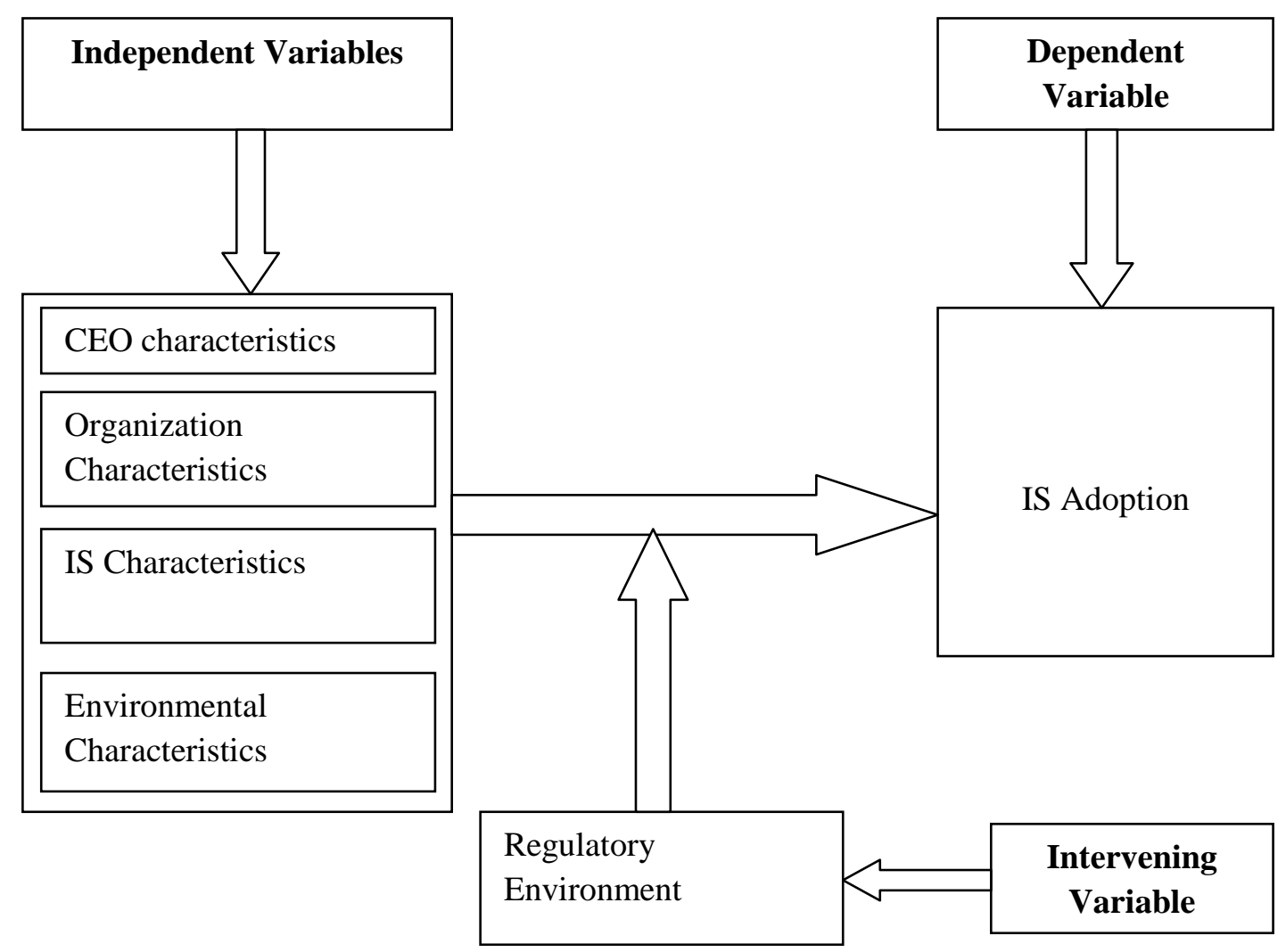

Figure 1: Conceptual Framework

Source: Researchers own

\subsection{Review of Related Literature}

\subsubsection{CEO Characteristics}

According to (Thong, 1999) the CEO Competency skills in Information technology and innovativeness are two attributes that influences IS Adoption in an organization. Lack of necessary skills and knowledge in information technology by a CEO of an organization will result into poor understanding of the need to deploy and adopt an IS in organization. CEOs innovative skills will also play a significant role in determining the level of IS Adoption and deployment by SMEs.

\subsubsection{Information System Characteristics}

Information System characteristics such as Relative advantage, compatibility, complexity, observability and trialibility influence the level of deployment and adoption of IS in an organization (Sahin I, 2005). Relative advantage of Information technology is the degree to which an innovation is perceived as being better than the idea it supersedes. For instance, the cost and social status motivation aspects of innovations are elements of relative advantage. Compatibility is the degree to which an innovation is perceived as consistent with the existing values, past experiences, and needs of potential adopters. According to (Thatcher, Foster, \& Zhu, 2006) lack of compatibility in ICT with individual needs may negatively affect the individual's ICT use. IS Complexity is the degree to which an innovation is perceived as relatively difficult to understand and use. IT Complexity is negatively correlated with the rate of adoption. Thus, excessive complexity of an innovation is an important obstacle in its adoption (Fincham et al., 1995). IS Trialibility is the degree to which an innovation may be experimented with on a limited basis. Trialibility is positively correlated with the rate of adoption. The more an innovation is tried, the faster its adoption is. IT Observability is the degree to which the results of an innovation are visible to others. Role modelling (or peer observation) is the key motivational factor in the adoption and diffusion of IS (Oracle, 2011).

\subsubsection{Organization Characteristics}

Business size, Employees Knowledge on Information technology and Information intensity are key factors that influence the level of IS deployment and adoption in an organization. According to (Chaffey, 2007) SMEs have highly centralized structures; tendency to employ generalists rather than specialists and financial resources makes SMEs to be unique. For instance, if SMEs wanted to recruit IS specialists, they face difficulties in attracting and retaining skilled IS staff because of the limited career paths available in a small business. As a result of this scenario, there is a lower level of awareness of the benefits of Information Systems and a lack 
of Information System knowledge and technical skill in SMEs. This hinders Information System adoption.

\subsubsection{Environmental Factors}

Competition in a business environment is likely to influence whether IS can be adopted or not in an organization. Competition increases the likelihood of innovation adoption in an organization. Other factors that influence Information Systems deployment and adoption include regulatory environment. According to (Van Grembergen, 2004), regulatory regime may influence the level of IS Adoption in an organization. Different countries have different legislative frameworks that may either promote or hinder Information systems deployment and adoption in an organization.

\section{METHODOLOGY}

The study used a quantitative descriptive survey design. Data was collected from 3 SMEs in TransNzoia County using Questionnaire. The study targeted 106 employees from Faulu Kenya, K-Rep Bank and Kenya Women Finance Trust in TransNzoia County. A sampling size of 36 respondents was chosen (Osoo \& Onen, 2008).

Table1: Table showing the Sampling Criteria Name of the SME Number of Sample Size Employees

\begin{tabular}{lcc}
\hline Faulu Kenya & 47 & 16 \\
K-Rep Bank & 33 & 11 \\
KWFT & 26 & 9 \\
Total & 106 & 36 \\
\hline
\end{tabular}

The sample size included CEO of three SMEs in TransNzoia County and other employees. The study employed both purposive and simple random sampling technique to identify the respondents that participated in the study. A structured questionnaire was used to collect data from the respondents. The CEO of the three SMEs was chosen to be the key informants in this study. The study identified the following variables

- CEOs Knowledge and Skills of Information Technology

- CEOs Innovativeness

- Competition

- $\quad$ Relative advantage of IS

- Compatibility of IS

- Complexity of IS

- $\quad$ Trialibility of IS

- $\quad$ Observability of IS

- Business Size

- $\quad$ Employees IS Knowledge

These variables were coded in $\mathrm{R}$ Version 3.0.0 software package. Both descriptive statistics and inferential statistics were used to analyse the data. Descriptive statistics used included use of histograms, frequency tables and pie charts to represent data. Inferential statistics was used to determine the relationship between the different variables as outlined in the research objectives.

All data was analysed at level significance of $95 \%$ or $\alpha=0.05$ and the degrees of freedom depending on the particular case as was determined.

\subsection{Findings}

Table 2: Table showing Descriptive Statistics

\begin{tabular}{lll}
\hline Variable & Mean & $\begin{array}{l}\text { Standard } \\
\text { Deviation }\end{array}$
\end{tabular}

\begin{tabular}{lcc}
\hline $\begin{array}{l}\text { CEOs Knowledge and Skills of } \\
\text { Information Technology }\end{array}$ & 2.400 & .58554 \\
CEOs Innovativeness & 1.8333 & .87831 \\
\hline Competition in the Industry & 1.3333 & .63246 \\
\hline Relative advantage of IS & 2.333 & .81064 \\
\hline Compatibility of IS & 2.411 & .86694 \\
\hline Complexity of IS & 2.411 & .62425 \\
\hline Trialibility of IS & 1.8222 & .84890 \\
\hline Observability of IS & 1.9556 & .80868 \\
\hline Business Size & 1.7778 & .89797 \\
\hline Employees IS Knowledge & 1.7500 & .90633
\end{tabular}

Variables measured on 1 to 5 scale

\section{Figure2: A pie chart showing the Descriptive Statistics}

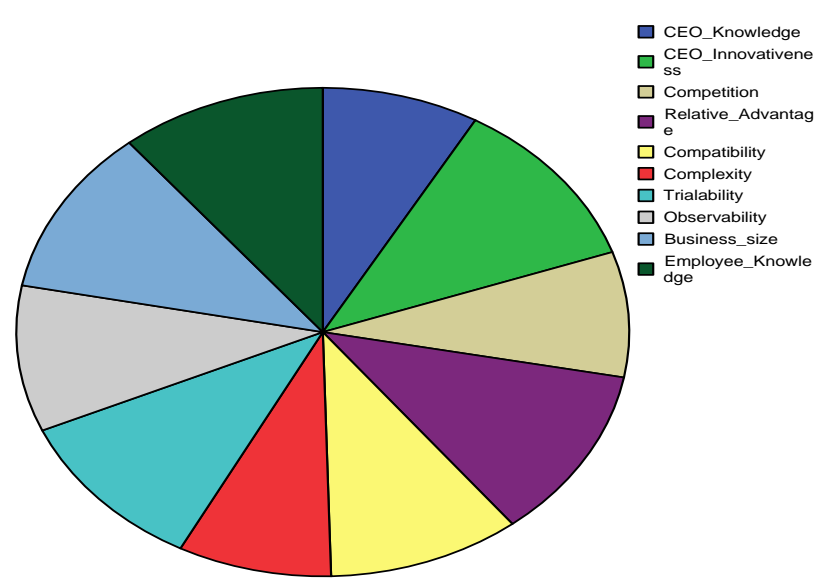


From the results, the following can be concluded that:

- $\quad 80 \%$ of the respondents cited CEO Knowledge and skills in IT as one of the major factors that influence the adoption and deployment of IS in an organization. This shows that CEOs Knowledge and skills in IT plays an important role in determining whether IS will be adopted or not by an organization.

- $60 \%$ of the respondents cited CEOs innovativeness as a determinant of IS adoption and Deployment in an organization. These shows that CEOs innovativeness in use of IT may contribute to the adoption and deployment of IT in an organization.

- Only $44 \%$ of the respondents cite Competition in the SME industry as a determinant of IS adoption and deployment in an organization.

- $75 \%$ of the respondents agree that Competitive advantage that IS provide influence the adoption and deployment IS in an organization. This reinforces Rogers Innovation Diffusion theory that identifies the IS characteristics as the main determinants of IS adoption.

- $81 \%$ of the employees cite Complexity of IS as a determinant of IS adoption and deployment in an organization. This reinforces Rogers Innovation Diffusion theory that identifies the IS characteristics as the main determinants of IS adoption.

- $\quad 78 \%$ of the employees cite trialibility as a determinant of IS adoption and deployment in an organization. This reinforces Rogers Innovation Diffusion theory that identifies the IS characteristics as the main determinants of IS adoption.

- $60 \%$ of the respondents cite observability as determinant of IS adoption and deployment in an organization. This reinforces Rogers Innovation Diffusion theory that identifies the IS characteristics as the main determinants of IS adoption.

- $60 \%$ of the respondents cited Business size as a determinant to the IS adoption and deployment in an organization. Majority of the respondents feel that the size of the business may influence the level of adoption. This may be due to financial constrains that face this institutions in installation of the latest technologies that may produce competitive advantage.

- $\quad 58 \%$ of the respondents cited employees IT Knowledge as determinant of IS adoption and deployment in an organization.

\section{CONCLUSIONS}

This paper examined the Determinants to Adoption and Deployment of Information Systems by SMEs in TransNzoia County, Kenya. The study found out that adoption and deployment of Information Technology is dependent upon the environment the IS will be deployed due to regulatory and environmental factors. The study found out that CEOs characteristics, IS characteristics, Organization characteristics and Environmental characteristics as the main determinants of IS adoption and deployment in an organization. However, these determinants may differ depending upon which environment is being referred to hence the different adoption levels in large corporations and SMEs. The study also found out that little research has been conducted in TransNzoia County, Kenya to ascertain the levels of IS adoption by SMEs hence the knowledge gap that the study sought to fill.

From the findings of the study, it was found that CEOs knowledge and skills in IT, IS relative advantage, Compatibility of IS, complexity of IS, Trialibility and Observability of IS are the highest contributors to IS adoption and deployment. The study highlights the importance of having innovative and IS-knowledgeable CEOs. From this findings it is also true that IS characteristics (relative advantage, Compatibility of IS, complexity of IS, Trialibility and Observability of IS) determines the adoption of IS in an organization. This reinforces Rogers Innovation Diffusion theory that identifies the IS characteristics as the main determinants of IS adoption (Sahin I, 2005). However, only $44 \%$ of the respondents cite Competition in the SME industry as a determinant of IS adoption and deployment in an organization. This contradicts other studies that found out that competition plays an important role in determining the adoption and deployment of IS in organizations. For instance, a study conducted by (Fincham et al., 1995)found out that competition plays an important role in determining IS adoption and deployment by SMEs in TransNzoia County, Kenya.

\section{ACKNOWLEDGMENTS}

I would like to thank my family: my wife Judith Orony and my son Troy Mulati for supporting me spiritually throughout my life. It is through their encouragement that I have accomplished this work. I sincerely thank them for their patience and understanding that gave me the peace of mind while conducting this study.

\section{REFERENCES}

[1] Barkley, markley, \& lamie. (2008). E-commerce as a business strategy: lessons learned from case studies of rural and small town businesses, 15 .

[2] CCK. (2010, December). ANALYSIS OF 2010 ICT SURVEY. CCK. Retrieved from http://cck.go.ke/resc/downloads/REPORT_OF_THE_NA TIONAL_ICT_SURVEY_2010.pdf

[3] Chaffey, D. (2007). E-business and E-commerce Management: Strategy, Implementation and Practice. Financial Times/Prentice Hall.

[4] Dien. (2002). E-business development for competitive advantages: a case study. Information \& Management 40 (2003) 581-590, 10.

[5] Fincham, R., Fleck, J., Procter, R. N., Scarborough, H., Tierney, M., \& Williams, R. (1995). Expertise and innovation: information technology strategies in the financial services sector. Oxford University Press. Retrieved from http://wrap.warwick.ac.uk/52966/

[6] Namisiko, P., \& Aballo, M. (2013). Current Status of eAgriculture and Global Trends: A Survey Conducted in TransNzoia County, Kenya. International Journal.

[7] Oracle. (2011). Commerce Anywhere: A Business and Technology Strategy to Maximize Cross-Channel Commerce Growth (p. 11). Presented at the ATG WEB COMMERCE, Oracle. 
[8] Osoo, W., \& Onen, D. (2008). A General Guide to Writing Research Proposal and Report (2nd Ed.). Makerere University: Makerere University Printery.

[9] S Jackson. (2011). Organization Culture and Information Systems Adoption. Journal of Organizational Behavior.

[10] Sahin I. (2005). Detailed review of Rogers' diffusion of innovations theory (Vol. 5).

[11] Thatcher, S., Foster, W., \& Zhu, L. (2006). B2B ecommerce adoption decisions in Taiwan: the interaction of cultural and other institutional factors. Electronic Commerce Research and Applications, 5(2), 92-104.

[12] Thong, J. Y. (1999). An integrated model of information systems adoption in small businesses. Journal of management information systems, 15(4), 187-214.

[13] TS Tran. (2011). Information Systems Adoption within Vietnam SMEs. Journal of Management Information Systems.

[14] Van Grembergen, W. (2004). Strategies for information technology governance. Igi Global. 